\title{
CAVITATION INCEPTION IN FLOW OVER A SQUARE-SECTION CYLINDER
}

\author{
M. M. Mosallem \\ Department of Mechanical Power Engineering and Energy; Faculty of \\ Engineering, Minia University, Minia, Egypt \\ E-mail: mosall2000@yahoo.com
}

(Received April 5, 2007 Accepted May 7, 2007)

This paper presents an experimental investigation of the onset of cavitation over a square-section cylinder. A numerical simulation of noncavitating steady incompressible turbulent flow around the cylinder was also performed. Three square-section cylinders of different sizes have been tested in a water tunnel. Cavitation inception has been detected visually. Observations of cavitation inception showed that the first visible traces of cavitation appear in the form of small bubbles in the free shear layer at the leading edge corners of the cylinders suggesting that the small-scale vortices in the shear layer over the side faces and associated pressure fluctuations are responsible for the onset of cavitation. The cavitation inception indices have been found to decrease with increasing the freestream velocity. In addition, the results showed that the variation of the cylinder size has no effect on the cavitation inception indices.

\begin{tabular}{|llll|}
\hline & \multicolumn{3}{c|}{ NOMENCLATURE } \\
$C p$ & Pressure coefficient & Greek symbols \\
$C p_{s}$ & Separation pressure coefficient & $\alpha$ & Total gas content, $\mathrm{ppm}$ \\
$H$ & Cylinder side length, $\mathrm{m}$ & $\varepsilon$ & Dissipation rate, $\mathrm{m}^{2} / \mathrm{s}^{3}$ \\
$k$ & Turbulent kinetic energy, $\mathrm{m}^{2} / \mathrm{s}^{2}$ & $\Phi$ & viscous dissipation term, $\mathrm{s}^{-2}$ \\
$p_{o}$ & Free stream pressure, $\mathrm{N} / \mathrm{m}^{2}$ & $\mu$ & Dynamic viscosity, N.s $/ \mathrm{m}^{2}$ \\
$p_{\text {min }}$ & Minimum pressure, $\mathrm{N} / \mathrm{m}^{2}$ & $\mu_{e}$ & effective viscosity, N.s $/ \mathrm{m}^{2}$ \\
$p_{s}$ & Separation pressure, $\mathrm{N} / \mathrm{m}^{2}$ & $\rho$ & Density, $\mathrm{kg} / \mathrm{m}^{3}$ \\
$p^{\prime}$ & Fluctuation pressure, $\mathrm{N} / \mathrm{m}^{2}$ & $\sigma_{i}$ & Cavitation inception number \\
$\operatorname{Re}_{H}$ & Reynolds number, & Subscripts \\
$\operatorname{Re}_{H}=\rho U_{o} H / \mu$ & $e$ & effective \\
$U_{o}$ & Free stream velocity, $\mathrm{m} / \mathrm{s}$ & $s$ & separation \\
& & min & minimum \\
\hline
\end{tabular}

\section{INTRODUCTION}

It is well known that cavitation inception occurs when a nucleus is exposed to a sufficiently low pressure to cause unstable growth and collapse of the cavity. Thus, the onset of cavitation is dependent on the availability of nuclei and the pressure of the flow field. Turbulent shear flows exhibit wide fluctuations in velocity and pressure, generally leading to a broad range of cavitation inception indices even for constant 
mean flow conditions. A turbulent shear layer contains primary (spanwise) and secondary (axial-streamwise) vortices (Bernal and Roshko [1] and Jimenez et al [2]). Numerous studies have focused on the relationship between these structures and cavitation. In small jets and small separated regions (Ooi and Acosta [3], Ran and Katz [4], Nakano et al [5], Belahadji, et al [6], and Mosallem [7]) cavitation inception occurs in the core of primary (small-scale) vortex structures that develop as the shear layer rolls up. However, as the size and the characteristic Reynolds number of the separated region increase, cavitation occurs in the secondary, axial vortices (Katz and O'Hern [8]; and O'Hern [9]). In these cases, cavitation extends into the large-scale primary structures only at ambient pressures that are considerably lower than the inception level. Gopalan et al [10] studied the cavitation inception in the near field of a jet. Cavitation inception was in the form of inclined 'cylindrical' bubbles. On tripping the boundary layer, cavitation inception occurred in the form of distorted 'spherical' bubbles. Baur and Köngeter [11] investigated the characteristics of the cavitation structures for flow over a rectangular sill fixed on the bottom of a channel with rectangular cross-section. It was observed that the cavitation structures have not a spherical but a developed longish form with the major axis at an angle of about $45^{\circ}$ towards the main flow. Recently, Wienken et al [12] developed a new method to predict cavitation inception for unsteady flows with traveling bubble cavitation. The method combines the enhanced predictive capabilities of large eddy-simulation (LES) with an algebraic stability criterion for the cavitation nuclei. The method was applied to the flow past a square cylinder. Both the predicted cavitation number as well as the location of cavitation inception are in good agreement with the experimental data. The cavitation inception was in the vortex cores in the wake of the cylinder.

Based on the review of the published data, the literature dealing with cavitation inception in separated flow over square-section cylinders are limited. Knowledge of cavitation inception in such flow is essential to get more information about cavitation phenomena in various turbulent separated flows. Therefore, the objective of the present work is to investigate in more details the physics of cavitation inception in the turbulent separated flow over square-section cylinders. The noncavitating flow around the cylinder's geometry was simulated in order to obtain details about the flow field.

\section{Computational details}

The flow is described in a Cartesian coordinate system, in which the $x$-axis is aligned with inlet flow direction as shown in Fig. 1. A fixed two-dimensional square-section cylinder with a side $H$ is exposed to a constant free stream velocity. An incompressible flow with constant fluid properties (water) is assumed. The flow around the cylinder was simulated using the Reynolds Averaged Navier-Stokes equations in conjunction with the $R N G k-\varepsilon$ turbulence model. The square-section cylinder side is $10 \mathrm{~mm}$ length. The form of the steady scalar transport equation is:

$$
\frac{\partial}{\partial x}(\rho u \phi)+\frac{\partial}{\partial y}(\rho v \phi)+\frac{\partial}{\partial z}(\rho w \phi)=\frac{\partial}{\partial x}\left(\Gamma_{\phi} \frac{\partial \phi}{\partial x}\right)+\frac{\partial}{\partial y}\left(\Gamma_{\phi} \frac{\partial \phi}{\partial y}\right)+\frac{\partial}{\partial z}\left(\Gamma_{\phi} \frac{\partial \phi}{\partial z}\right)+S_{\phi}
$$

Where:

$$
\begin{aligned}
\Gamma_{\phi} & =\text { diffusion coefficient } \\
S_{\phi} & =\text { source term }
\end{aligned}
$$




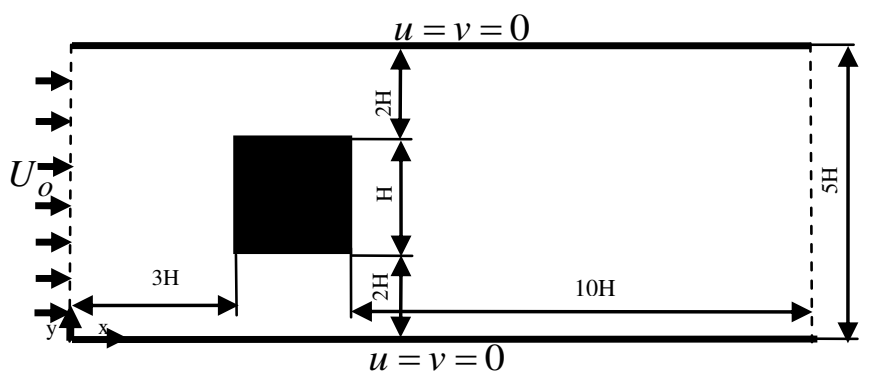

Fig. 1. Computational domain for the calculation of the flow over square-section cylinder.

Table 1 shows the variables, coefficients, and source terms for the transport equations

\begin{tabular}{|l|l|l|l|}
\hline$\phi$ & Equation & $\Gamma_{\phi}$ & $S_{\phi}$ \\
\hline 1 & Mass conservation & 0 & 0 \\
\hline$u$ & x-momentum & $\mu_{e}$ & $\rho g_{y}-\partial p / \partial y$ \\
\hline$v$ & y-momentum & $\mu_{e}$ & $\rho g_{x}-\partial p / \partial x$ \\
\hline$k$ & Turbulent kinetic energy & $\mu_{t} / \sigma_{k}$ & $\mu_{t} \Phi-\rho \varepsilon$ \\
\hline$\varepsilon$ & Dissipation rate & $\mu_{t} / \sigma_{\varepsilon}$ & $C_{1} \mu_{t} \varepsilon \Phi / k-C_{2} \rho^{2} / k$ \\
\hline
\end{tabular}

Where

$$
\begin{aligned}
& \mu_{e}=\text { effective viscosity } \\
& \Phi=\text { viscous dissipation term } \\
& C_{1}, C_{2}, \sigma_{k} \text { and } \sigma_{\varepsilon} \text { are the } R N G \text { model coefficients }
\end{aligned}
$$

The following boundary conditions are used:

- The inlet is placed some body heights upstream of the body, where $u=U_{o}$ and $v=0$ is prescribed

- No slip-conditions are prescribed at the cylinder surface and duct walls, $u=v=0$

- At the outlet of the domain atmospheric pressure is assumed

The governing equations were solved using a segregated velocity-pressure solution algorithm where the momentum equation is used to generate an expression for the velocity in terms of the pressure gradient. This is used in the continuity equation after it has been integrated by parts. A grid of 84714 nodes was enough to predict the flow. This finding was drawn from comparison of the computed mean pressure coefficient, $C_{p}$ along the cylinder surface for different grids with each other. The results showed that further refinements would not greatly alter the predicted flow features. 


\section{EXPERIMENTAL SETUP AND EXPERIMENTAL TECHNIQUES}

The experiments were conducted in the test section of the water tunnel described schematically in Fig. 2. The tunnel consists of a closed water circuit. The test section (1) is $500 \mathrm{~mm}$ long and its cross-section is $25 \mathrm{~mm}$ x $50 \mathrm{~mm}$. The four sides of the test section are perspex for photographic and observational purposes. Velocity can be varied by using a brass gate valve. In addition, the pressure can be changed by varying the air pressure at the top of the partial filled resorber tank. The fresh water has been used as a working fluid. All tests have been conducted at room temperature (nominally $\left.30{ }^{\mathrm{O}} \mathrm{C}\right)$.

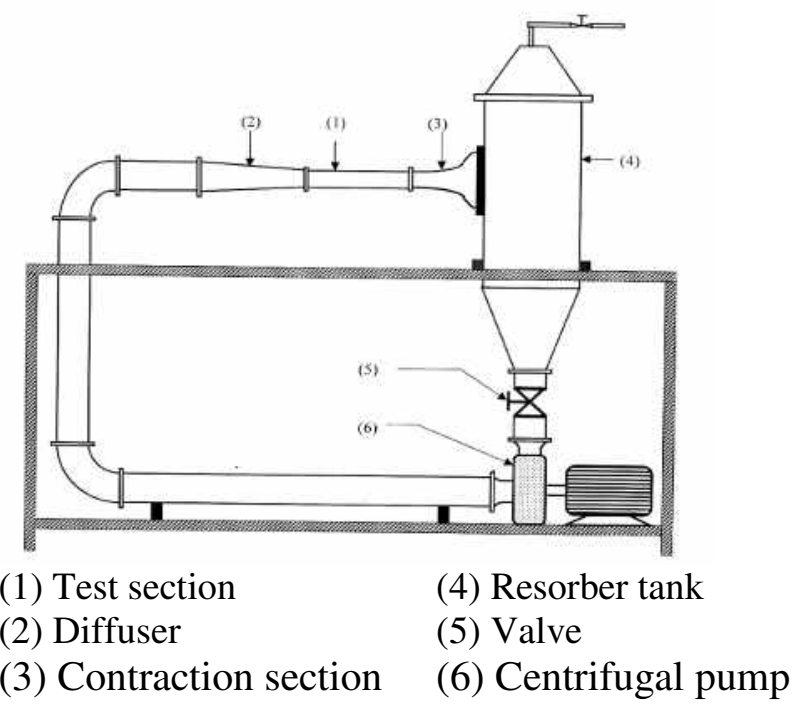

Fig. 2. Schematic diagram of the water tunnel

Three square-section cylinders with sides of length, $H$ of 5, 10 and $12 \mathrm{~mm}$ were used during the course of the investigation. In the experiments described here the models were mounted with two faces parallel with the direction of upstream flow. The static-pressures have been measured at two locations on lower side face of the $10 \mathrm{~mm}$ side cylinder. Static-pressure holes drilled in the surface of the cylinder are connected to mercury manometer boards by means of plastic tubes. The free stream water static pressure has been measured with a U-tube mercury manometer. The velocity in the test section has been measured by using the measured value of pressure difference across the contraction section with a mercury manometer. This has been achieved by calibrating the contraction section using a pitot tube.

Cavitation inception has been detected visually by noting the occurrence of a few minute bubbles, namely when cavitation was already macroscopic. The measurement procedure involved establishing the tunnel pressure fixed, then increasing the speed until cavitation events occurred. The cavitation inception is defined in this investigation as the onset of detectable discrete bubbles which first appear, as described by Huang [13]. The cavitation number, $\sigma$ is given by $\sigma=\left(p_{o}-p_{v}\right) / 0.5 \rho U_{o}^{2}$ and the cavitation inception index is denoted by $\sigma_{i}$. The 
cavitation aspects were photographed simultaneously with the corresponding flowmeasurements using a digital camera. The photos were later computer processed and printed. The dissolved gas content has been measured by a dissolved gas content meter, which is based on the principle of Van Slyke, (Van Slyke and Neill [14]).

\section{RESULTS AND DISCUSSION}

\section{Computational results}

In this section, the results obtained from the steady state computations of flow around the square-section cylinder are presented. The computed time-averaged streamlines around the cylinder at $\operatorname{Re}_{H}=5.65 \times 10^{4}$ (where $\operatorname{Re}_{H}$ is the Reynolds number based on cylinder side length, $H$ and freestream velocity, $U_{o}$ ) are shown in Fig. 3. As shown, the flow separates at the leading edge corners of the cylinder, and the near wake of the cylinder is characterized by the formation and shedding of large-scale vortices. In the figure two vortices are clearly seen. The first one is being shed away from the cylinder while the second one is still attached and growing. This pattern is similar to that reported by Rodi et al [15].

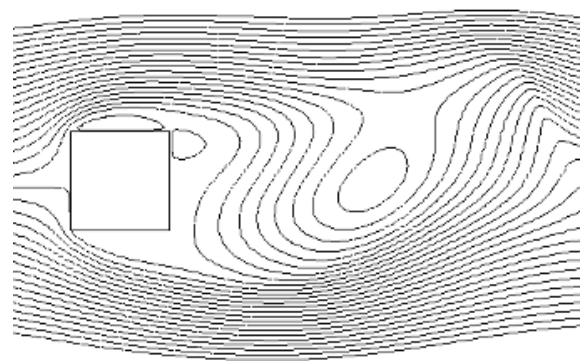

Fig. 3. Time average streamlines around the square-section cylinder

$$
\left(H=10 \mathrm{~mm}, \operatorname{Re}_{H}=5.65 \times 10^{4}\right)
$$

In order to determine the domain of nuclei activation, the pressure contours around the cylinder were plotted in Fig. 4. It can be observed that separated region over the side faces of the cylinder surface and the vortex cores in the near wake are the regions of low pressures.

Figure 5 shows the computed pressure coefficient around the square-section cylinder surface. The trend is similar to that predicted numerically by several authors (as reported by Rodi et al [15]) and that obtained from measurements presented by Bearman and Obasaju [16].

\section{Experimental Results}

As mentioned earlier, the static-pressures have been measured at two locations on lower side of the $10 \mathrm{~mm}$ side cylinder. Fig. 6 shows a comparison between the calculated and experimental surface pressure distributions. It can be observed that the measured pressure at the two locations on the lower surface is fairly well predicted. 


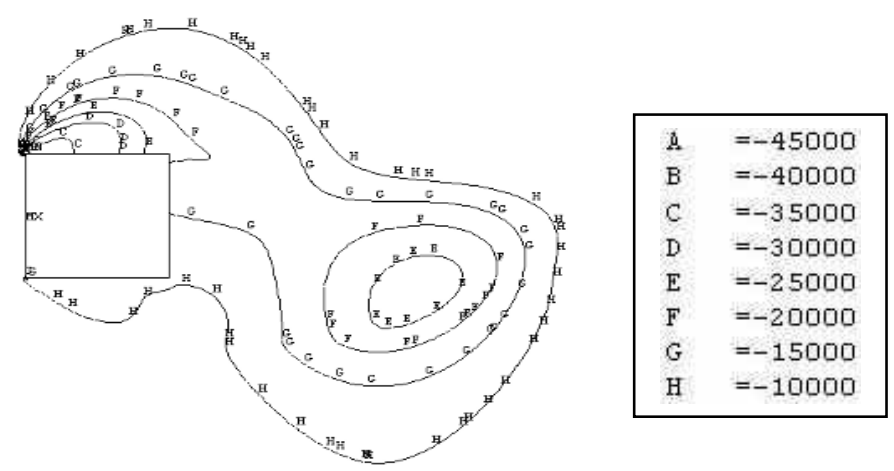

Fig. 4. Computed pressure contour behind the square-section cylinder

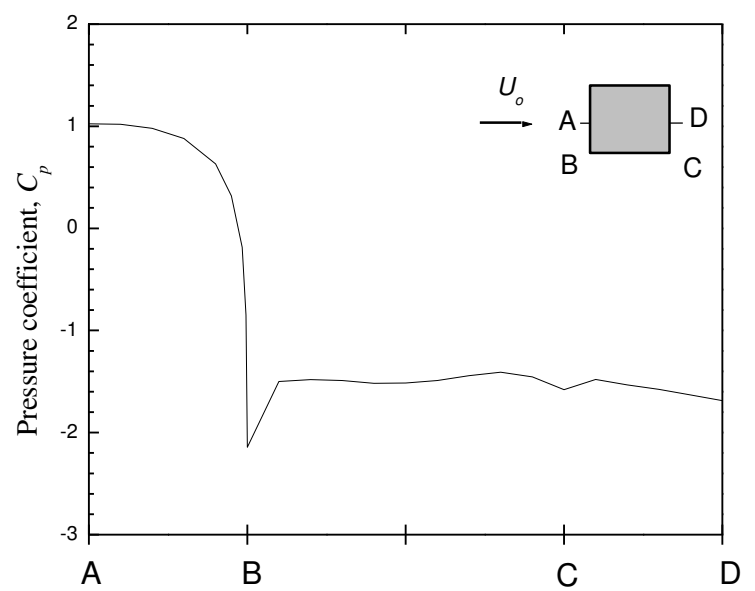

Fig. 5. Computed pressure coefficient around square-section cylinder $\left(H=10 \mathrm{~mm}, \operatorname{Re}_{H}=5.65 \times 10^{4}\right)$

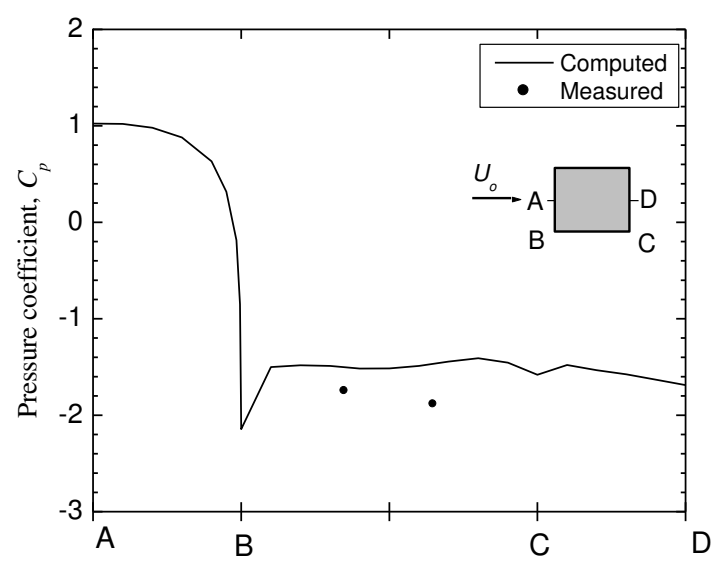

Fig. 6. Comparisons between the calculated and experimental surface pressure distributions $\left(H=10 \mathrm{~mm}, \operatorname{Re}_{H}=5.65 \times 10^{4}\right)$

The experiments of cavitation inception have been made by lowering the cavitation number until the cavitation began to appear. The physical appearance of 
cavitation at inception was in the free shear layer over the side faces at the leadingedge corners of the cylinder. Bearman and Obasaju [16] measured the fluctuating pressures around square-section cylinder. It has been found that root-mean square values of the pressure fluctuations on the side faces are larger than those on the front and rear faces. This suggested that the small-scale vortices shedding from the leadingedge corners of the cylinder and associated pressure fluctuations are responsible for beginning of cavitation inception on the side faces of the cylinder. A similar type of cavitation had been observed in the small-scale vortices behind a backward facing step (Mosallem [7]) and in the near wake of a wedge (Belahadji [6]). As mentioned previously, Wienken et al [12] observed that the cavitation at inception was in the vortex cores in the wake of the cylinder. In the present work the cavitation inception indices were obtained by beginning with initial tunnel pressures under the atmospheric pressure. As indicated by Arndt et al. [17] when the test section pressure was reduced below the saturation value, the volume of free gas increased markedly and the nuclei sizes will be larger. Also the turbulence has a pronounced effect on the cavitation inception, Rood [18]. Therefore, the disagreement between the cavitation inception location observed in the present study and that observed by Wienken et al [12] may be related to the difference in both initial nuclei sizes and the turbulence intensity.

When reducing the cavitation number from inception value, the number of bubbles on the side-faces increased. As the cavitation number decreases, the cavitation region grows to cover the side faces of the cylinder, Figs 7 to 9 . Also the video images revealed the vortex shedding behind the cylinder where the vortex was visualized by the cavitation bubbles. A further reduction in the cavitation number results in the occurrence of fully developed cavitation where the cavitation bubbles filled the entire near wake region as shown in Figs 10 to 12. The results of the inception and developing of cavitation for the three cylinders were similar. The total gas content, $\alpha$ as measured by the gas content apparatus varied between 16 and $17 \mathrm{ppm}$.

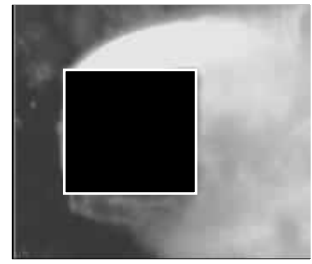

Fig. 7. Cavitation over square-section cylinder, $U_{o}=8.1 \mathrm{~m} / \mathrm{s}, \sigma=2.11, H=5 \mathrm{~mm}$

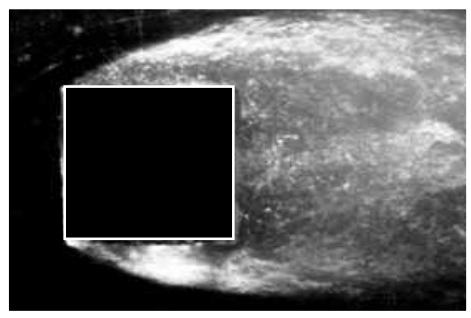

Fig. 9. Cavitation over square-section cylinder, $U_{o}=6.6 \mathrm{~m} / \mathrm{s}, \sigma=3.57, H=12 \mathrm{~mm}$

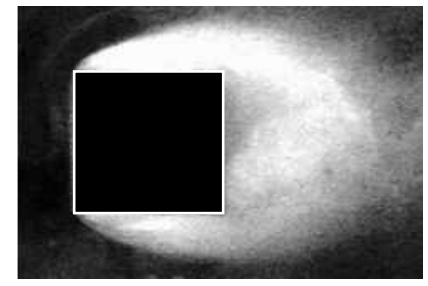

Fig. 8. Cavitation over square-section cylinder, $U_{o}=7.4 \mathrm{~m} / \mathrm{s}, \sigma=2.65, H=10 \mathrm{~mm}$

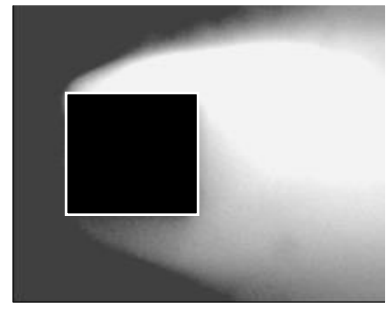

Fig. 10. Cavitation over square-section cylinder, $U_{o}=9.3 \mathrm{~m} / \mathrm{s}, \sigma=1.35, H=5 \mathrm{~mm}$ 


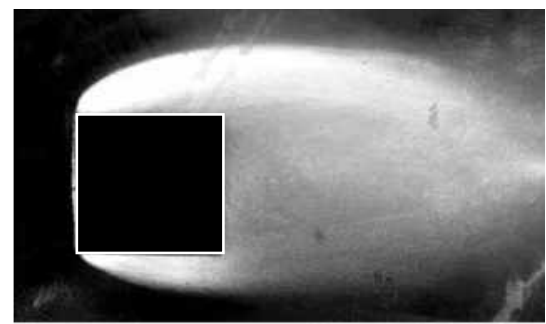

Fig. 11. Cavitation over square-section cylinder, $U_{o}=9.8 \mathrm{~m} / \mathrm{s}, \sigma=1.36, H=10 \mathrm{~mm}$

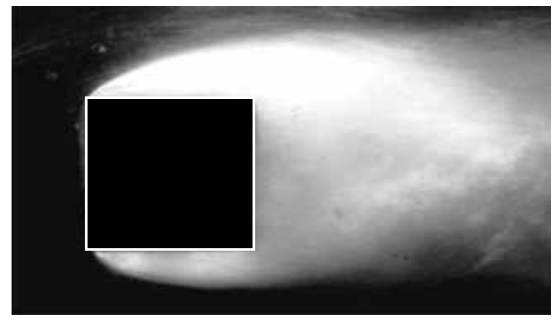

Fig. 12. Cavitation over square-section cylinder, $U_{o}=7.3 \mathrm{~m} / \mathrm{s}, \sigma=2.76, H=12 \mathrm{~mm}$

The cavitation inception indices for each cylinder obtained at various free stream velocities are displaced in Figs 13 to 15 respectively. Clearly, it can be observed that the cavitation inception indices for the three cylinders have approximately the same trend. The cavitation inception indices decrease with increasing of free stream velocity. A similar trend has been noticed by Mosallem [7] for flow past a backward facing step. In addition, Ran and Katz [4] for a turbulent jet found that the inception indices tend to decrease with increasing velocity. It was also observed that the sites of the earliest traces of cavitation agree with the location of highest negative pressure fluctuations peaks. They demonstrated that although the root-mean-square values of pressure fluctuations do not vary with the jet speed, the magnitudes and likelihood of high negative pressure peaks decreases with increasing velocity, causing a reduction in the inception index with increasing velocity

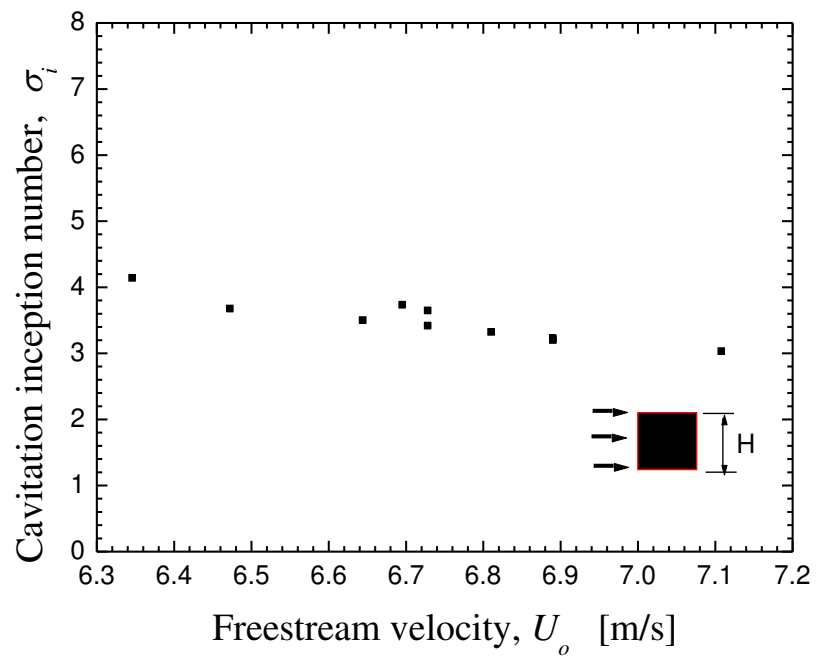

Fig. 13. Cavitation inception indices for square-section cylinder,

$$
H=5 \mathrm{~mm}
$$




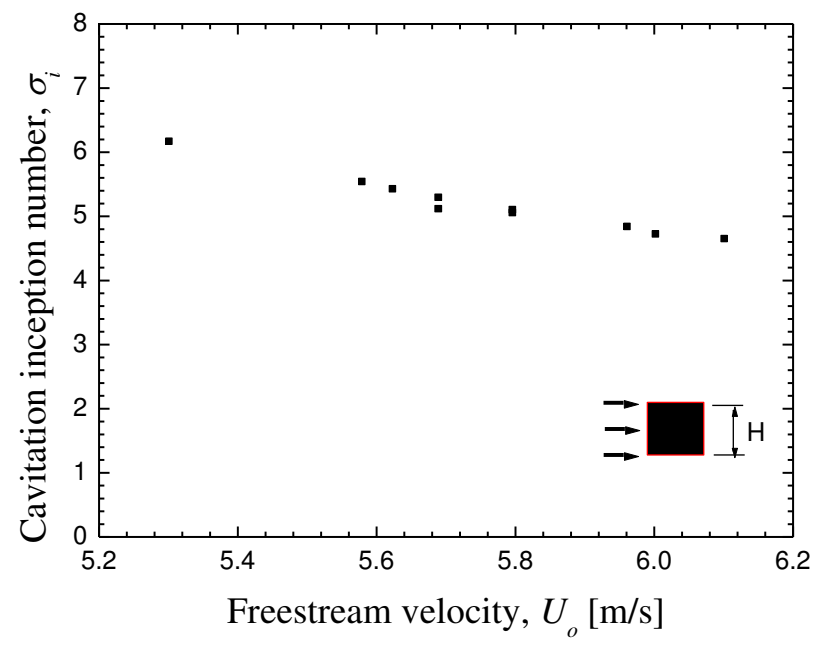

Fig. 14. Cavitation inception indices for square-section cylinder, $H=10 \mathrm{~mm}$

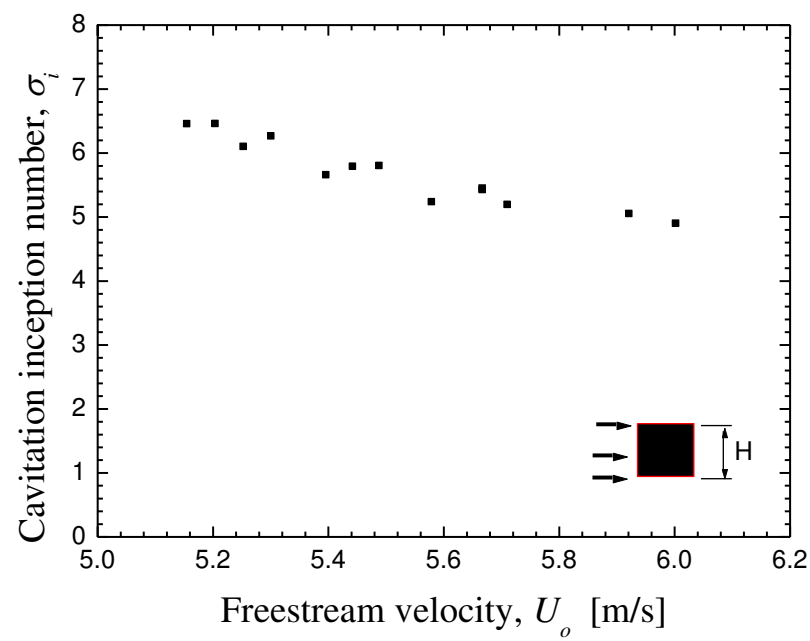

Fig. 15. Cavitation inception indices for square-section cylinder,

$$
H=12 \mathrm{~mm}
$$

It is also evident that the measured values of cavitation inception indices of the present study are large. As reported by O'Hern [19] the mean pressure variations in a turbulent shear flow are often small, but the instantaneous peak pressures vary significantly from the mean. These pressure peaks are responsible for the relatively large cavitation inception indices commonly measured in turbulent shear flows. The large values of inception indices show that inception occurs under mean pressures much larger than the vapor pressure, but do not provide any information on the response of a nucleus to the localized pressure peaks it is exposed to. This supports our opinion that the cavitation inception in the shear layer over square-section cylinder 
may be related to the small scale vortices and associated fluctuating pressure peaks in the shear layer at the leading-edge corners of the cylinders.

In Fig. 16 the cavitation inception data for the three square-section cylinders were replotted to obtain a clear comparison. It is evident that the cylinder size has no effect on the cavitation inception indices. This means that within the tested range of the cylinder size and freestream velocities, the pressure fluctuations are independent on the cylinder size.

\section{Modeling of cavitation inception}

As presented by Cimbala and Billet [20] and Arakeri et al [21], the cavitation occurs when the minimum pressure, $p_{\text {min }}$ equals the vapor pressure. The cavitation inception number can be expressed in the form:

$$
\sigma_{i}=\left(p_{o}-p_{\min }\right) / 0.5 \rho U_{o}^{2}
$$

The minimum pressure can be written as

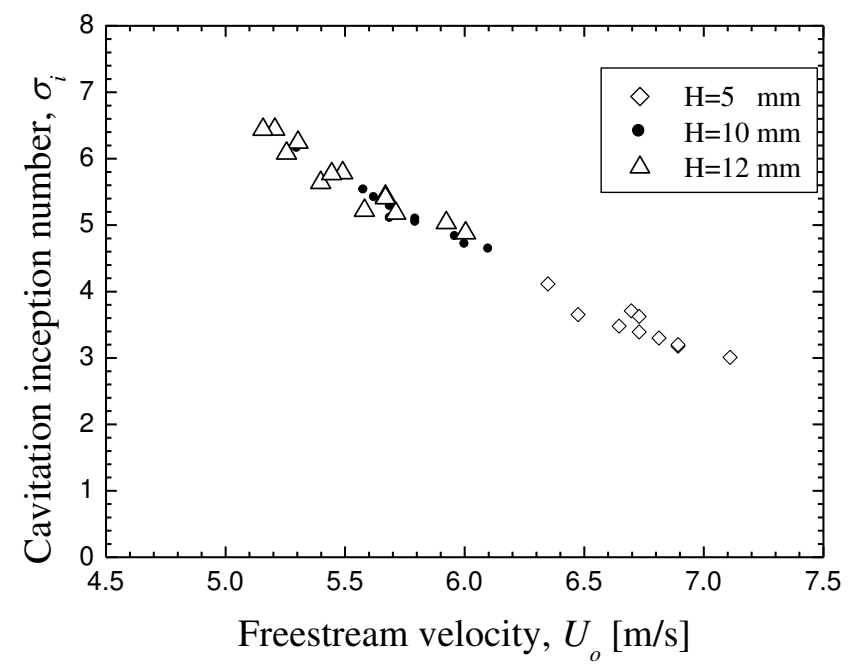

Fig. 16. Effect of cylinder size on the cavitation inception indices

$$
p_{\text {min }}=p_{s}-p^{\prime}
$$

where $p_{s}$ is the mean separation pressure at the cylinder leading edges and $p^{\prime}$ is the turbulent fluctuation pressure below the mean value.

Employ Eq. (3) in Eq. (2) yields that:

$$
\begin{aligned}
& \sigma_{i}=\left(p_{o}-p_{s}\right) / 0.5 \rho U_{o}^{2}+p^{\prime} / 0.5 \rho U_{o}^{2} \\
& \sigma_{i}=-C p_{s}+p^{\prime} / 0.5 \rho U_{o}^{2}
\end{aligned}
$$

Thus, the cavitation inception number can be predicted if the values of both separation pressure and the fluctuating pressure are known. 


\section{CONCLUSIONS}

In the present study, the physics of onset of cavitation over square-section cylinders have been investigated experimentally. The flow field around the cylinder was predicted numerically. Cavitation inception indices using visual criterion are presented over a range of tunnel speeds for three square-section cylinders of different sizes. The observations of cavitation inception showed that the cavitation inception appears in the form of small bubbles in the free shear layer at the leading edge corners of the cylinders. Therefore, the small-scale vortices in the shear layer over the side faces and associated pressure fluctuations are responsible for the onset of cavitation. Furthermore, the cavitation inception indices have been found to decrease with increasing the freestream velocity. In addition, the results showed that the variation of the cylinder size has no effect on the cavitation inception indices.

\section{REFERENCES}

[1] Bernal, L. P. \& Roshko, A. "Streamwise vortex structure in plane mixing layers" J. Fluid Mech., 170, 499, (1986).

[2] Jimenez, J., Cogollos, M. and Bernal, L. P. "A perspective view of the plane mixing layer" J. Fluid Mech., 152, 125, (1985).

[3] Ooi, K. K. and Acosta, A. J. "The utilization of specially tailored air bubbles as static pressure sensors in a jet" Trans. ASME, J. Fluids Engng., 106, 459, (1983).

[4] Ran, B. and Katz, J. "Pressure fluctuations and their effect on cavitation inception within water jets", FED-Vol. 177, Cavitation Inception, ASME, 31, (1993).

[5] Nakano, k., Hayakawa, M., Fujikawa, S. and Yano, T. "Cavitation bubbles in a starting submereged water jet", CAV2001: $4^{\text {th }}$ International Symposium on Cavitation, California Institute of Technology, Pasadena, California, USA, 20-23 June, (2001).

[6] Belahadji, B., Michel, J.M. and Franc, J.P. "Cavitating vortices in the turbulent wake of a wedge", $2^{\text {nd }}$ international symposium on cavitation, Tokyo, Japan, April, 51, (1994).

[7] Mosallem, M. M. "Bubble dynamics and cavitation inception in a turbulent separating flow" $4^{\text {th }}$ International Symposium on Experimental and Computational Aerothermodynamics of Internal Flows, Dresden, Germany, August 31 to September 2, (1999).

[8] Katz, J. and O'Hern, T.J. “Cavitation in large scale shear flows" Trans. ASME, J. Fluids Engng., 108, 373, (1986).

[9] O'Hern, T. J. "An experimental investigation of turbulent shear flow cavitation" J. Fluid Mech., 215, 365, (1990).

[10] Gopalan, S., Katz, J. and Knio, O. "The flow structure in the near field of jets and its effect on cavitation inception' J. Fluid Mech., 398, 1, (1999).

[11] Baur, T. and Köngeter, J. "The three-dimensional character of cavitation structures in a turbulent shear layer" XIX IAHR International Symposium on Hydraulic Machinery and Cavitation, Singapore, 9-11 September (1998). 
[12] Wienken, W., Stiller, J. and Keller, A. "A method to predict cavitation inception using large-eddy simulation and its application to the flow past a square cylinder", Trans. ASME, J. Fluids Engng., 128, 316, (2006).

[13] Huang, T.T. "The effects of turbulence stimulators on cavitation inception of axisymmetric headforms", Trans. ASME, J. Fluids Engng., 108, 261, (1986).

[14] Van Slyke, D.D. and Neill, J.M. "The determination of gases in blood and other solutions by vacuum and manometric measurements", J. of Biological Chemistry, 61, 523, (1924).

[15] Rodi, W., Ferziger, J.H., Breuer, M. and Pourquié, M. "Status of large eddy simulation: results of a workshop", Trans. ASME, J. Fluids Engng., 119, 248, (1997).

[16] Bearman, P.W. and Obasaju, E.D. "An experimental study of pressure fluctuations on fixed and oscillating square-section cylinders", J. Fluid Mech., 119, 1, (1982).

[17] Arndt R.E.A., Arakeri, V.H. and Higuchi, H. "Some observations of tip-vortex cavitation", J. Fluid Mech., 229, 269, (1991).

[18] Rood, E., "Review-Mechanism of Cavitation Inception," Trans. ASME, J. Fluids Engng., 113, 163, (1991).

[19] O'Hern, T.J. "Cavitation inception scale effects; I. Nuclei distributions in natural water, II. Cavitation inception in a turbulent shear flow ", Ph.D. Thesis, California Institute of Technology, Pasadena, California, May 4 (1987).

[20] Cimbala, J.M. and Billet, M.L. "Cavitation inception in rectangular slots", International symposium on cavitation inception, FED. ASME., 89, 35, (1989)

[21] Arakeri, V.H., Carroll, J.A. and Holl, J.W. "A note on the effect of short and long laminar separation bubbles on desinent cavitation", Trans. ASME, J. Fluids Engng., 103 , 28, (1981).

\section{بدء التكهف في سريان فوق أسطوانة مريعة المقطع}

هدف هذا البحث هو دراسـة بدء التكهف في سريان فوق اسطوانة مربعة المقطع. فقد تم دراسة بدء التكهف في هذا السريان معطليا، كما تم عمل محاكاة عددية للسريان حول الأسطوانة. وقد تم اختبار

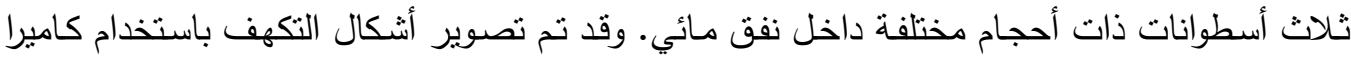
رقميـة. وقد أوضـت التجارب المعمليـة أن بدء التكهف كان عبارة عن ظهور فقاعات صغيرة عند الحافتين الأماميتين للأسطوانة مما بشير إلى أن هذه الفقاعات تكونت نتيجة للضغط المنخفض في قلب

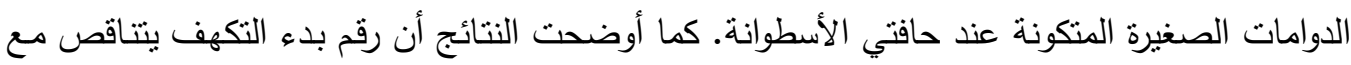

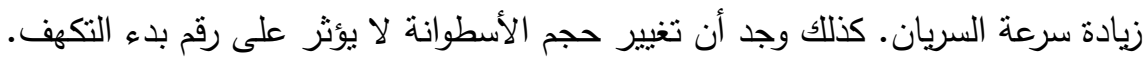

